\title{
Impacts of the Inclusion of Distributed Generation on Congestion of Distribution Networks and in the Islanding Operation Capability
}

\author{
Jose David Beltrán Gallego; Leidy Daniela Castro Montilla; Alexandra Castro Valencia; Camilo Augusto Giraldo \\ Muñoz; Dahiana López García \\ DOI: https://doi.org/10.32397/tesea.vol2.n2.3 \\ Research paper
}

Received: 15 August 2021; Accepted: 10 December 2021; Published: 15 December 2021

\begin{abstract}
The growing demand for electricity in the world has led to power systems having to constantly increase their generation capacity and expand their transmission and distribution systems. Consequently, distributed generation has positioned as a technology able to integrate generation close to consumption centers, freeing up capacity in the transport systems, which can be translated into a deferral of investments in network expansion. Therefore, this paper analyzes the impact of the inclusion of distributed generation in the congestion of a typical distribution network and evaluates the potential of providing the island operation capability ancillary service in a section of the system to identify the possible challenges and benefits that the development of this technical support service could have in typical Colombian distribution networks.
\end{abstract}

Index Terms-Distributed Energy Resources; Distributed Generation; Distribution Network; Islanding Operation; Microgrids, Network Congestion.

\section{NOMENCLATURE}

\begin{tabular}{ll}
\hline Acronym & Definition \\
\hline CHP & Combined Heat and Power \\
DG & Distributed Generation \\
DER & Distributed Energy Resources \\
SHP & Small Hydro Power \\
$w_{i}$ & Weighting factor for the ith line \\
$I_{n_{i}}$ & Nominal capacity of the ith line \\
$m_{a x}\left(I_{n}\right)$ & The highest rated capacity \\
$N_{L}$ & Number of lines in the system \\
$C F_{i-k}$ & The congestion factor of the ith line for the kth period \\
$T C F_{k}$ & Total system congestion for the kth period \\
$N_{k}$ & Number of periods in the evaluation horizon \\
\hline
\end{tabular}

Jose David Beltrán Gallego, Universidad Nacional de Colombia sede Manizales, (email: jbeltrang@unal.edu.co)

Leidy Daniela Castro Montilla, Universidad Nacional de Colombia sede Manizales, (email: ledcastromo@unal.edu.co)

Alexandra Castro Valencia, Universidad Nacional de Colombia sede Manizales, (email: alcastrova@unal.edu.co)

Camilo Augusto Giraldo Muñoz, Universidad Nacional de Colombia sede Manizales, (email: cagiraldomu@unal.edu.co)

Dahiana López García, Universidad Nacional de Colombia sede Manizales, (email: dahlopezgar@unal.edu.co)

\section{INTRODUCTION}

$\mathbf{I}$ $\mathrm{N}$ recent decades, society has advanced rapidly, and therefore, the electricity sector has had to quickly adapt to a series of changes and challenges related to the increase in energy demand, energy security, and climate change [1]. This energy transition has generated a trend towards the introduction of local electricity generation in distribution networks through the use of conventional and renewable energy sources such as natural gas, biogas, wind energy, photovoltaic solar units, fuel cells, combined heat and power (CHP) systems, microturbines and Stirling engines [2], to meet these needs and ensure an affordable, clean, reliable and efficient power supply.

This local generation is known as Distributed Generation (DG), which together with energy storage systems and responsive demand are define as Distributed Energy Resources (DERs) [1]. Integration of DG in distribution networks changes the operating paradigm of these networks, transforming passive networks with unidirectional information and power flow into active networks that recognize the importance of monitoring and control in real-time and with bi-directional power flows [3]. The implementation of DG can influence both positively and negatively on the operation of the network depending on the characteristics under which both the distribution system and the DG operate. The positive impacts are generally related to the capacity to provide ancillary services such as voltage control and the improvement in power quality, the reduction of losses, and the release of capacity in the transmission and distribution network [4]. Among these services stands out the island operating capability, this capability is a condition in which a microgrid with DG is isolated electrically from the rest of the network due to a failure that occurs in the central supply network or due to any other disturbance that potentially affects the continuous supply to users, whether momentary or permanent [3].

The implementation of these microgrids capable of supplying the island operation capability ancillary service seeks to solve the need for a more flexible, efficient, and reliable electrical distribution system while reducing the environmental impact and the congestion of the network and encouraging demand participation [5], [7]. Nevertheless, for a system that operates in island mode to be able to provide the above- 
mentioned benefits and be sustainable in a long-term scenario, the operating guidelines must be adapted to the context of the network, so it is necessary to analyze and determine the technical parameters of the assets immersed in the network, the energy potentials of the zone, the behavior of the connected users, and the regulatory policies that apply to each particular case [6], [8].

In this sense, this paper evaluates the impact of the inclusion of distributed generation in the congestion of a Colombian typical distribution network, while analyzing the potential for providing the island operation capability ancillary service in a portion of the system, identifying the possible challenges and benefits that the development of this technical support service could have in typical distribution networks.

\section{MICROGRID OPERATION}

A system with the capacity to operate by islands due to the inclusion of DERs is characterized by being an electrical power network with the integration of distributed energy resources and control systems. This grid can be an isolated network or part of a local electrical network that can be disconnected and reconnected to the central electrical system according to adequate previously planning and without negatively affecting the technical parameters of the principal network. Its implementation brings with it impacts in the electrical system, depending on several elements related to the regulatory and technical conditions (focused on the design, performance, and operation of the electrical system, as well as its architecture) [9].

The global technical guidelines to carry out an efficient and reliable design of a microgrid with the integration of DERs focus on different factors such as the characteristics and functionality of the distributed resources, changes in the magnitude and direction of the power flow, the adequate voltage and frequency control, power quality, the existence of one or more coupling common points, local generation system or load requirements, steady-state and transient conditions, interactions between different electrical power sources present in the system, reserve margins, demand response, cold load activation 11 , monitoring, control, information exchange, and protection schemes [2], [9].

To guarantee the satisfactory operation of an electric power system with integration of DG when it begins to operate as an island some factors must be considered, such as the risk of reconnection out of phase, the negative implications in assets due to anomalies in voltage or frequency, the reliability of the local network with increased system complexity, possible reduction in power quality, changes in fault currents between normal and island operating modes, the coordination of the system protections, coordination with load shedding schemes, the voltage and frequency control schemes, and load or angular phase shift [9].

Another important technical aspect is the reconnection of the island or microgrid to the power system. During this

\footnotetext{
${ }^{1}$ Overcurrent condition that occurs when a distribution circuit is reenergized after a prolonged outage.
}

process, emphasis should be placed on monitoring the synchronization and closing of protection elements due to differences in voltage, frequency, and phase between the microgrid and the central system must be minimal and within acceptable limits. This reconnection process can occur through different methods such as active and passive synchronization and de-energized island [3].

\section{A. Limitations, barriers, and benefits of microgrids operation}

To achieve microgrids effective deployment in modern energy systems, they must overcome several technical, regulatory, economic, and social problems. On the other hand, some standards and regulations promote the integration of DERs into the electricity grid; but, only a few specifically address the integration of microgrids, so adequate regulatory support is necessary for these [5].

For safe operation, the microgrid transition between connected mode and island operation mode must not lead to service interruption or stability problems; also, the microgrid must guarantee adequate power quality, frequency, and voltage for consumers. An additional challenge is to reach an agreement between all the stakeholders immersed in the microgrid operation, that is, the community, the network operator, the government, and the owners of the generators [5].

As above-mentioned, if the integration of DERs in the distribution networks is not properly managed, some adverse effects can arise in the system, such as the sudden transition to connection mode to the central system when operating in island mode during momentary or permanent faults. The last can lead to an overvoltage in the load, an overload of the generators immersed in the microgrid, or the complete disconnection of the system, which is remarkably undesirable. Another factor is that low inertia loads and generators are the big problems to maintain the stable operation of the island, and if users have cogeneration facilities following the thermal load curve, or if the primary resources are non-controllable renewable sources such as wind or solar generation, there are some aspects to mention: low energy quality, the possibility of limited production and operation under conditions of energy limitation due to the variability of the primary resource [10].

The main operational and management problems related to microgrids' operation are [5]: (A) Power quality must be maintained in the short term through the proper balance of active and reactive power, (B) the microgrid must operate isolated in regions where the supply of public services is not available; therefore, it must be able to take advantage of local energy resources, (C) Planning of the generation, supply, and storage of energy needs to consider the load curve of the microgrid and the long-term energy balance since any unbalance between generation and demand can lead the system to complete disconnection, (D) Contingency analysis and planning of emergency operation are essential to maintain the security of supply (such as demand-side management, load shedding, island or shutdown of any unit), (E) In contingency conditions, an economic reprogramming of the generation must be performed to maintain continuous supply at least at the priority loads, and to maintain the voltage and frequency of 
the load between operational limits, and (F) adequate telecommunications infrastructures and communication protocols must be employed for the management, protection, and control of the microgrid.

However, the benefits of a microgrid can be twofold: the local benefits resulting from the internal microgrid's operation, and the broader benefits derived from how the microgrid interacts with the central system. Additional local advantages include increased energy reliability and continuity of supply for customers inside and outside the microgrid, and the reduction of the congestion of the distribution network; and the benefits of indirect microgrid operation are broader, but also more difficult to quantify and are typically related to the release of capacity in the central system, and the provision of ancillary services from the microgrid to the principal network [11], [12].

Microgrids help reduces stress on the transmission network by sharing their loads [5]; however, the existing transmission and distribution networks were not designed to accommodate distributed generation, consequently, in many regions, the network operators expect an increase in network congestion due to the integration of these resources, especially in rural and isolated areas. In distribution networks, the uncontrolled integration of these DERs can result in voltage increases, a rise in fault currents, a decrease of system inertia, and the need for re-coordination of the protection scheme. In transmission, the transfer of a large volume of energy from areas with a lot of DG to load centers can cause transport congestion through the networks and lead to the need to limit the power coming from DG to avoid the energy not supplied and the permanent elongation of the lines [13].

Therefore, the sustainable planning of microgrids can result in a potential solution to reduce overloads and congestion of transmission lines. Then, when properly planned, a microgrid has the potential to alleviate the congestion of the bulk power system in which it is integrated by reducing the power import from the central network and can increase the stability of the system to respond to rapid changes in demanded or generated power, maintaining supply quality to customer satisfaction. Its planned integration also allows the deferral of investments in the expansion of the transmission system and the addition of peak generation units [5].

Therefore, microgrids, such as active low-voltage and medium-voltage networks, can potentially provide lots of benefits to the central system by increasing the efficiency and continuity of supply of distribution networks and improving the reliability, continuity, and quality of service [5].

\section{B. Operation by intentional islands}

The term intentional or planned island consists of the separation of the DG with its assigned demand. These microgrids have been developed to increase reliability, reduce losses, decrease costs, and diversify the electricity supply. The intentional island is not risky as its disconnection and reconnection to the central electrical grid is planned according to the required needs. For operation on the intentional island mode, it is necessary to carry out several analyzes, studies, and simulations on the selected feeder at different demands and operating conditions. Several factors must be analyzed, such as fault currents, the internal inertia of the island, the voltage profiles, the coordination of the existing protection equipment, and other operating parameters [14].

The most valuable benefits of DG include improved security of supply, reduced greenhouse gas emissions, congestion decrease, reduced transport energy losses, and increased investment flexibility. Consequently, beyond the several advantages that conventional power systems provide, the following benefits have led to the gradual development and integration of DG systems [2]: (A) Due to the rapid growth of the load, the need to increase conventional generation causes a continuous depletion of the fossil fuel reserve. Therefore, most of the countries are looking for unconventional renewable energy resources as an alternative. (B) The reduction of environmental pollution and global warming acts as a fundamental factor to prefer renewable resources to fossil s. Exploiting DERs is expected to help generate environmentally friendly clean energy with a much lower environmental impact. (C) The DERs' independent and grid-connected operations help increase generation capacity, thus improving overall power quality and reliability. Furthermore, a deregulated environment and open access to the distribution network also offer more opportunities for DG integration.

\section{CASE OF STUDY}

In this section, there is a description of the selected network and the simulations developed in this study, as well as a brief explanation of the methodology proposed to analyze the system congestion seeking to verify that with the adequate immersion of distributed resources it is possible to reduce congestion in the system and, in addition, to demonstrate the impacts of the operation island.

\section{A. Proposed network}

The configuration of the network used to carry out the analysis of this study was the IEEE 37-bus system (Fig. 1) because its characteristics correspond to a typical distribution network with a radial topology that allows representing the immersion of DERs in systems of this type. The base scenario of this network does not have the connection of generation sources since it is a passive network and depends entirely on the external network; also, it has an installed load of 2,735 MVA and a voltage of $4.8 \mathrm{kV}$ [3].

The demand curve used for the simulations of this system (Fig. 2) was gathered from the data recorded in the official demand forecast of XM SA ESP corresponding to October 2020 in the Andean region and with behavior typical regarding the Colombian demand [16].

To simulate the integration of variable generation, a photovoltaic generation unit is connected to the 710-bus as suggested by [3], [17], since it has one of the lowest voltages of the system in stable operation. After choosing the buses for the immersion of the distributed generation unit, it is necessary to analyze the characteristic solar irradiance curve of the area and thus approximate its behavior. 


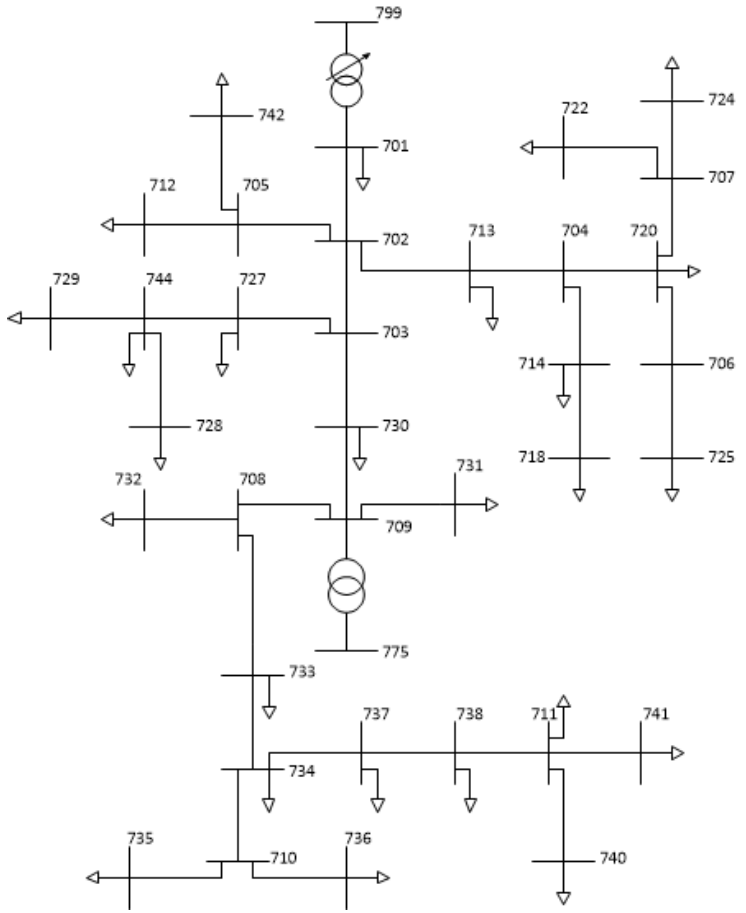

Fig. 1. IEEE 37 node test feeder with an installed load of 2,735 MVA and a voltage of $4.8 \mathrm{kV}$. Source: Own elaboration based on [15].

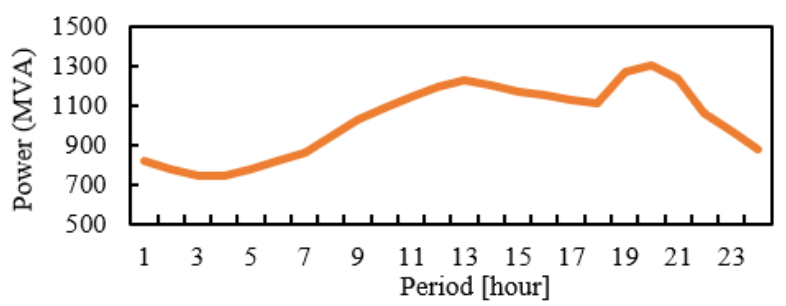

Fig. 2. Average demand curve October of 2020 in the Andean Region. Source: Own elaboration based on [16].

The solar generation curve can be seen in Fig. 3 and corresponds to the average solar generation of a unit located in the Colombian Andean region for October 2017. The data had been scaled for the considered case of study, where the photovoltaic generation unit has a nominal power of $500 \mathrm{~kW}$.

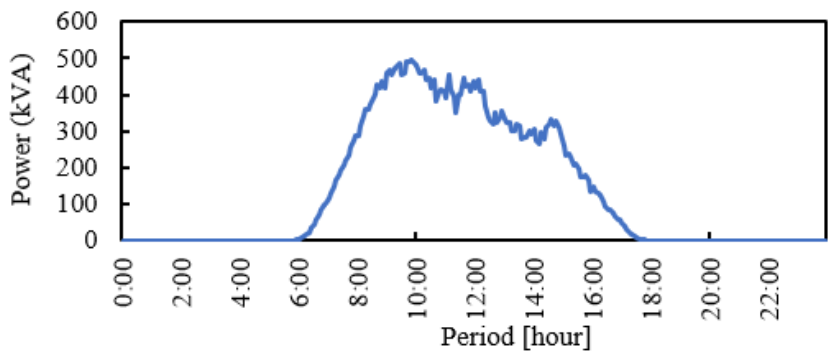

Fig. 3. Solar irradiance curve of a photovoltaic solar system within the Andean region. Source: [18].

To simulate the inclusion of distributed generation in the system, one synchronous generation plant with a capacity of 1,5 MVA is integrated; this represents a Small Hydro Power
(SHP) since the water resource is the most abundant in the region. This plant is selected because it has the maximum power demanded by the net load within the intentional island that makes up the downstream area of bar 709. This synchronous generation unit is connected to the 711-bus, which meets the same considerations as bar 710 concerning voltage in the passive operation scenario [3], [17]. Furthermore, it is necessary to clarify that 709-bus, where the intentional island is formed, has been suggested and used in previous studies [3].

The principal analysis developed is a congestion study for evaluating the impact of DG integration into a typical distribution system. This analysis will also allow identifying the potential of the island operation mode of a section of the network when there is the integration of distributed generation. The following scenarios are used from now on to obtain results and analyze them:

- Scenario 1: The system operates as a passive network without the presence of DG, then it is expected to act according to the typical behavior of a distribution network, where the sole determining factor of the congestion is the demand curve.

- Scenario 2: The system operates as an active network with the presence of the described DG units.

- Scenario 3: For this case, the system operates in intentional island mode, working in isolation from the principal network. This island is composed of the bars, lines, loads, and generation units located downstream of the 709 bus.

\section{B. Proposed methodology for the study of system congestion.}

The proposed methodology evaluates the congestion in the system each period of the time horizon. Traditionally, the total congestion calculation methods for electric power systems consider the marginal nodal prices of each system-nodes, which cannot be calculated for this case of study due to the absence of generation units in the baseline scenario. Consequently, the following procedure is performed to calculate the total system congestion in each period of the time horizon.

First, a priority factor for all system lines is calculated following (1).

$$
w_{i}=\frac{I_{n_{i}}}{\max \left(I_{n}\right)}, \quad i=1, \cdots, N_{L}
$$

where:

- $w_{i}$ is the weighting factor for the ith line.

- $I_{n_{i}}$ is the nominal capacity of the ith line.

- $\max \left(I_{n}\right)$ is the highest rated capacity among all lines in the system.

- $N_{L}$ is the number of lines in the system.

Then, for each period of the time horizon, the congestion of each line of the system is determined. For this, the line congestion is weighted with the factor calculated in (1), and finally, according to (2), the sum of the weighted congestions of all lines for each evaluation period is performed. 


$$
T C F_{k}=\sum_{i=1}^{N_{L}} C F_{i-k} \cdot w_{i}, \quad k=1, \cdots, N_{k}
$$

Where:

- $T C F_{k}$ is the total system congestion for the kth period.

- $C F_{i-k}$ is the congestion factor of the ith line for the kth period, which is obtained by dividing the current of the ith line in the kth period by its nominal capacity.

- $N_{k}$ is the number of periods in the evaluation horizon.

\section{RESULTS AND ANALYSIS}

To perform the congestion analysis in the system, two priority factors were taken into consideration.

\section{A. Priority factor 1: Complete system}

This priority factor was calculated considering all the lines of the system; therefore, it only applies for the scenarios one (1) and two (2).

\section{- Scenario 1: passive distribution system}

For this case, total congestion of the system is 91,06 , where the most congested hours are between 7:00 p.m. and 9:00 p.m.; This is the typical behavior expected for a passive network in which the congestion will naturally follow the trend of the demand curve since the power consumption is the only determinant of the current flow in the lines, this is reflected in Fig. 4.

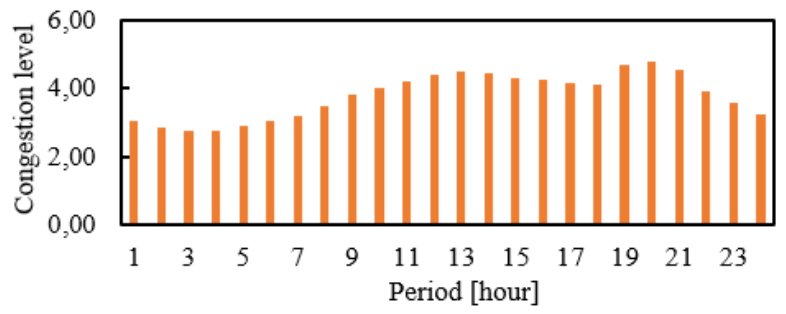

Fig. 4. Congestion in the system in each period of the time horizon for the first scenario and the priority factor one (1). Source: Own elaboration.

\section{- Scenario 2: active distribution system}

The inclusion of the photovoltaic generation unit makes the total congestion in the system to be reduced to approximately 58.40, which represents a reduction of $35.86 \%$. It can be seen in Fig. 5 that this case follows the same behavior as the previous one, but with the difference that the first peak is less pronounced, and all levels of congestion decreased when compared to the one obtained for the first scenario.

Fig. 6 shows that when making a comparison between congestion levels for the two cases discussed above, it is evident that for the second scenario, congestion is minor in each of the evaluated periods, even reaching a $42 \%$ decrease compared to the initial congestion at 1:00 p.m. The most significant effect could be observed between 12:00 and 16:00 hours, because when the first peak in demand occurs, simultaneously a photovoltaic solar generation peak is originated, achieving that the generation

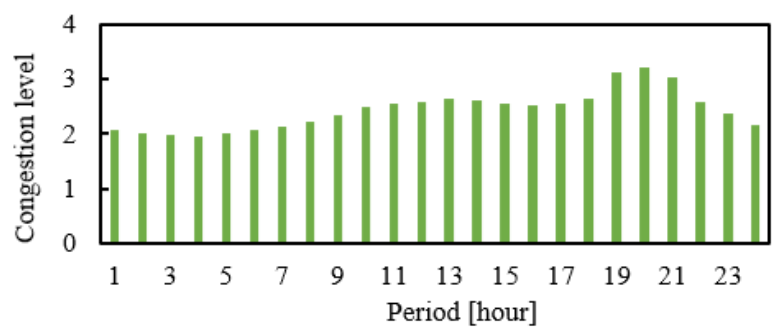

Fig. 5. Congestion in the system in each period of the time horizon for the second scenario and the priority factor one (1). Source: Own elaboration.

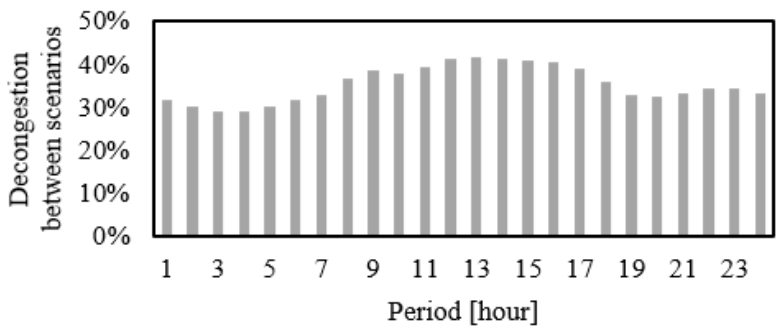

Fig. 6. Decongestion percentage of the second scenario compared to the base case (first scenario) for all periods of the evaluation horizon and the priority factor one (1). Source: Own elaboration.

by distributed sources immersed on the network reduces congestion significantly in this period.

One of the elements that allow having a better perception of the effect that the immersion of DG will have in the system is the behavior of congestion on the lines, where the idea is that the congestion will not increase with the inclusion of DG.

When carrying out this verification, the overall congestion in the system decreases since most of the lines have a reduced congestion level in the active distribution system network scenario, with only congestion increases in the lines 734-710, 734-737, 737-738, and 738-711, the last two being the most critical.

The lines of this distribution network were conceived to transport the energy from the external network to loads of the system, i.e., in a downstream direction of the 734bus, so the previous result is expected according to the location of DG because the downstream load of the 734bus is small compared to the total load of the system, leading the DG to satisfy said demand and then export energy to the rest of the network, thus congesting these lines since they communicate the GD with the 734-bus which is the one that leads the current to the rest of the network. In this way, there is more significant congestion on lines 737-738 and 738-711 because they communicate the SHP with the rest of the network, being this DG the one with the highest capacity.

On the other hand, the results obtained from the analysis of the system voltages in each of the scenarios reflect that: when operating passively, the maximum voltage value in the network is 1.0 p.u., and the minimum is 0.957 p.u., and when operating actively due to the inclusion of DG these values change respectively to 1.006 p.u. and 0.978 
p.u., presenting a better network operation since the range in which the voltage varies daily is reduced.

\section{B. Priority factor 2: Island region}

To obtain more telling results of the effects that are generated in the system when operating in island mode, it will be necessary to calculate a new priority factor only considering the lines of the system in the region defined downstream of the 709 bar and then, with this, redo the congestion calculation for all scenarios.

\section{- Scenario 1: passive distribution system}

In this case, the congestion in the system region follows a trend comparable to the one of the demand curves; the global congestion is 56.79, and the highest congestion level is found between 7:00 p.m. and 9:00 p.m., as can be seen in Fig. 7.

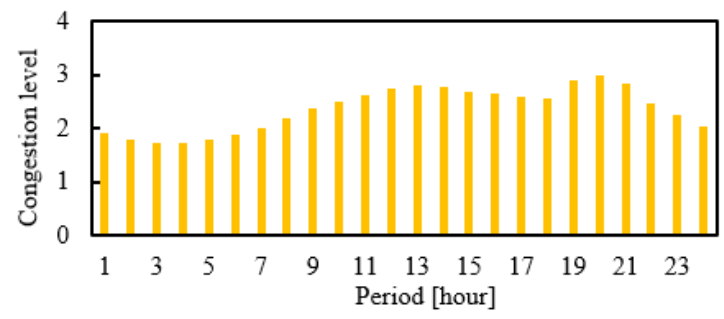

Fig. 7. Congestion of the portion of the network to be operated on the island for all periods of the evaluation horizon for the first scenario with the second priority factor. Source: Own elaboration.

\section{- Scenario 2: active distribution system}

With the immersion of DG in bars 710 and 711 , the overall congestion level decreases to a value of 38.23 , reducing approximately $32.68 \%$. Fig. 8 shows that the most marked peak of congestion occurs between 10:00 and 11:00 hours which is a consequence of the inclusion of the DG since the lines located near it tend to increase its congestion. Additionally, the network that is upstream of 709-bus has a power flow supplied by the external network, which affects the lines that are between 709bus and 710-bus.

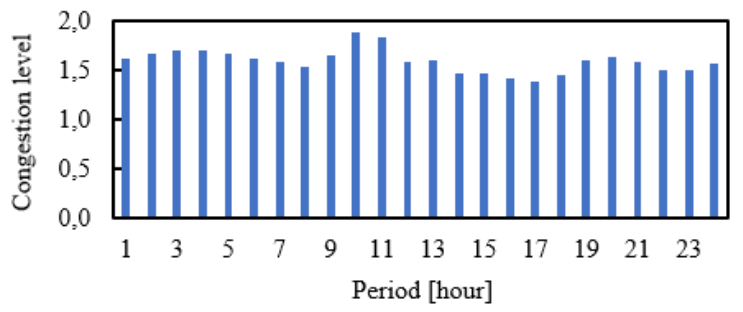

Fig. 8. Congestion of the portion of the network to be operated on the island for all periods of the evaluation horizon for the second scenario with the second priority factor. Source: Own elaboration.

The island region's voltage under passive distribution system fluctuates between 0.985 p.u. and 0.957 p.u. and for active distribution system between 1,006 p.u. and 0.982 p.u., demonstrating that the inclusion of DG improves the voltage corresponding to the region of the island because suitable nodes were selected for the integration of DG.

- Scenario 3: island operation mode

When the microgrid (formed downstream 709-bus) operates isolated from the principal network (opening the switch between bars 709 and 730), a reduction in overall system congestion is achieved, compared to the previously analyzed scenarios, having a global congestion value of 36.89 .

Fig. 9 highlights the appreciable reduction in the island's congestion between 9:00 a.m. and 5:00 p.m., compared to the first scenario, due to the photovoltaic generation unit, which provides a significant power injection in this interval as shown in Fig. 3.

Finally, between 7:00 p.m. and 9:00 p.m., there is the peak with the highest congestion, which is due to the response to the demand curve of the system, in which the total load of the island is higher which requires a greater current through the system lines, which translates in an increase of the network congestion.

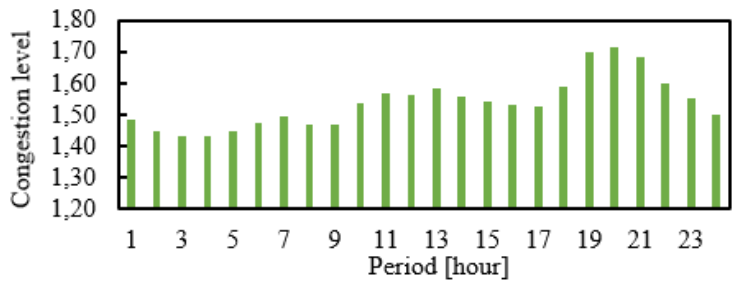

Fig. 9. Congestion of the portion of the network to be operated on the island for all periods of the evaluation horizon for the third scenario and the second priority factor. Source: Own elaboration.

Fig. 10. shows the percentual decongestion generated by DG integration into the system, mainly when photovoltaic generation injects power into the grid. Also, when photovoltaic generation does not supply part of the grid demand, the SHP unit helps to Decongest the system throughout the analyzed time-horizon, mainly during the peak demand between 7:00 p.m. and 9:00 p.m. Analogously, in Fig. 11. the same behavior is presented comparing the passive network and island operation mode.

Also, it is essential to note that congestion levels in some lines tend to increase with the inclusion of DG, mainly lines 737-738 and 738-711 because they are closer to the synchronous generation unit, which is the one providing the most power to the island region.

Finally, Fig. 12 shows the observed decrease in network congestion for the island operation mode in the instants when the demand curve is not close to its peaks; this is because the SHP doesn't have to saturate the surrounding lines. On the other hand, when the demand is close to its peaks, an increase in congestion is observed, which is reflected in the change of sign since congestion becomes higher compared to the active network scenario since there is no power supply from the external network and the SHP unit finds it necessary to inject more power 
to be able to satisfy the demand, thus congesting the surrounding lines.

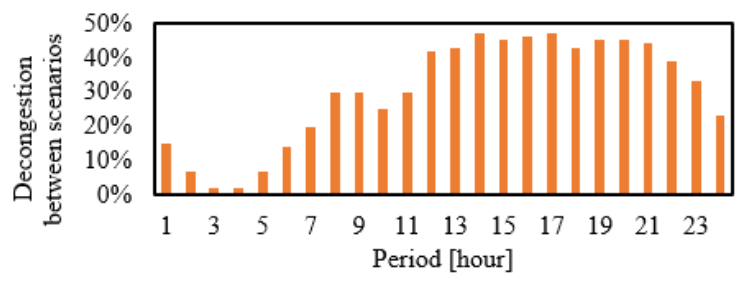

Fig. 10. Decongestion percentage of the second scenario compared to the base case (first scenario) for all periods of the evaluation horizon with the second priority factor. Source: Own elaboration.

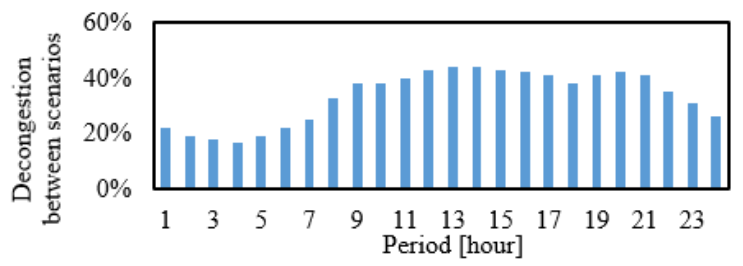

Fig. 11. Decongestion percentage of the second scenario compared to the base case (first scenario) for all periods of the evaluation horizon with the second priority factor. Source: Own elaboration.

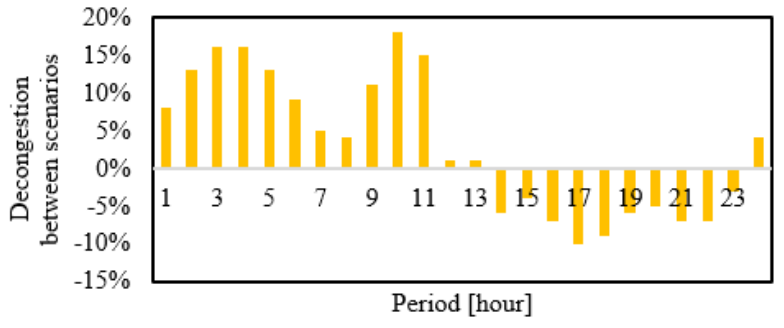

Fig. 12. Decongestion percentage of the third scenario compared to the second scenario for all periods of the evaluation horizon with the second priority factor. Source: Own elaboration.

The voltage for this scenario show high variability in the voltages of the buses that belong to the island; for example, at 10:00 a.m., there are overvoltages in all the buses of the island because there is a peak of photovoltaic generation, for this reason, it is suggested to carry out previous studies to determine an adequate voltage control system. On the other hand, between 5:00 p.m. and 11:00 p.m., the voltage are below the $5 \%$ allowed by the regulation (and even the 10\% allowed for rural areas) due to the absence of photovoltaic solar generation and the high demand that occurs at these moments, which forces the SHP unit to increase its power injection, causing a decrease in the reactive generation capacity (due to its capability curve), which affects the system voltage.

The protection scheme is another aspect essential to be considered in the island mode operation since the integration of DG changes the distribution of current flows in the grid and may vary the short-circuit power flow, leading to potential challenges in the coordination scheme [19]. Therefore, it is necessary to look for new alternatives that recognize the bidirectional power flows and the topology changes of the active power flow compared to the traditional operation of passive distribution grids. Some potential solutions have been suggested by literature such as directional protections, fault current limiters, protection schemes based on fuzzy logic, among others [19], [20].

\section{CONCLUSIONS}

In this paper, a congestion analysis was developed for a typical distribution network when DG units are connected to the system, at the same time as evaluating the possibility that this generation allows a section of the distribution network to operate as an intentional island if there is a fault in the central grid.

The analysis developed made it possible to identify that a good location of the DG can reduce the overall congestion of the system, nevertheless, it is necessary to consider the capacity of the lines that communicate these generation assets with the rest of the network since the power injection of these generation units may increase the congestion of adjacent lines, and can even cause over currents that would reduce the reliability and quality of the supply, and can lead to having trapped generation.

In the intentional island operation of the network, it was possible to establish that isolated mode operation generates a reduction in the congestion of the lines of the system, thus freeing up capacity in the principal supply network.

The results obtained for the active operation of the network allow to verify that the integration of DG units in the appropriate nodes allows to improve the voltage profile of the system, so the development of optimal location studies before the introduction of these units in the system is suggested.

It was possible to verify that, although with the integration of distributed generation units in the distribution networks emerges the possibility of operating sections of these grids as intentional islands, it is necessary to perform additional studies for the successful provision of the island operation capability ancillary service. This because it is crucial to search for voltage control mechanisms that allow maintaining this parameter within the established operational ranges when operating in island mode. At the same time, the implementation of a frequency control scheme that allows responding to sudden changes in power generated by variable generation units such as photovoltaic solar units is necessary, since in this study an average solar generation curve was considered for the PV unit, but the daily behavior can present marked change gradients that can significantly reduce the inertia of the system and can lead the network to complete disconnection.

Future work must study the protection schemes for the correct operation in island mode because this paper only focuses on the capacity supplied by the DG in case the island portion of the grid is disconnected from the central network.

\section{REFERENCES}

[1] F. P. Sioshansi, Smart Grid - Integrating Renewable, Distributed \& Efficient Energy, Menlo Ener. 2012. 
[2] S. Chowdhury, S. P. Chowdhury, and P. Crossley, Microgrids and active distribution networks. 2009.

[3] J. A. González, "Análisis técnico para la implementación de la capacidad de operación por isla a partir de la generación distribuida proveniente de cogeneración," Universidad Nacional de Colombia, sede Manizales Facultad, 2020.

[4] H. D. Mathur, "Enhancement of power system quality using distributed generation," PECon2010 - 2010 IEEE Int. Conf. Power Energy, pp. 567-572, 2010, doi: 10.1109/PECON.2010.5697646.

[5] J. D. Mina-Casaran, D. F. Echeverry, and C. A. Lozano, "Demand response integration in microgrid planning as a strategy for energy transition in power systems," IET Renew. Power Gener., vol. 15, no. 4, pp. 889-902, 2021, doi: 10.1049/rpg2.12080.

[6] J. D. Marín Jiménez, "Análisis para la provisión del Servicio Complementario de Capacidad de Operación por Islas a partir de Recursos Energéticos Distribuidos en ambientes desregulados," Universidad Nacional de Colombia, 2017.

[7] J. D. Marín-Jiménez, S. X. Carvajal-Quintero, and A. Arango-Manrique, "Discusión de la implementación en Colombia del servicio complementario capacidad de operación por islas," Energética, vol. 0, no. 43, pp. 99-108, 2014.

[8] S. P. Chowdhury, S. Chowdhury, and P. A. Crossley, "UK scenario of islanded operation of active distribution networks with renewable distributed generators," Int. J. Electr. Power Energy Syst., vol. 33, no. 7, pp. 1251-1255, 2011, doi: 10.1016/j.ijepes.2011.01.004

[9] IEEE Standards Coordinating - Committee 21, IEEE Guide for Design, Operation, and Integration of Distributed Resource Island Systems with Electric Power Systems, no. July. 2011.

[10] A. O. Egorov, S. A. Eroshenko, V. O. Samoylenko, P. V. Kolobov, and D. A. Glushkov, "Island Mode of Low Capacity Generators Operation," Adv. Mater. Res., vol. 1008-1009, pp. 426-429, Aug. 2014, doi: 10.4028/www.scientific.net/AMR.1008-1009.426.

[11] O. Palizban and K. Kauhaniemi, "Microgrid control principles in island mode operation," in 2013 IEEE Grenoble Conference, Jun. 2013, pp. 1-6, doi: 10.1109/PTC.2013.6652453.

[12] D. López-García, "Caracterización de un esquema remunerativo para la participación de la demanda en la prestación del servicio complementario de control de frecuencia en el mercado eléctrico colombiano," 2019.

[13] J. Munsch, "Formación intencional de islas en sistemas eléctricos de potencia con generación eólica," 2014.

[14] P. F. Vásquez Miranda and N. R. Fabara Tobar, "Propuesta para lograr la Operación en Isla Intencional de un Sistema real de Distribución que dispone de Generación Distribuida," in XXVII Jornadas en Ingeniería Eléctrica y Electrónica, 2017, vol. 27, pp. 201-208

[15] IEEE Distribution System Analysis Subcomitte, "IEEE 37 NodeTest Feeder." 1992.

[16] XM SA ESP, "Pronostico oficial de demanda," 2020. https://www.colibri.udelar.edu.uy/jspui/handle/20.500.12008/5208.

[17] J. C. Hernández, A. Medina, and F. Jurado, "Optimal allocation and sizing for profitability and voltage enhancement of PV systems on feeders," Renew. Energy, vol. 32, no. 10, pp. 1768-1789, Aug. 2007, doi: $10.1016 /$ j.renene.2006.11.003.

[18] Universidad Nacional de Colombia: Grupo de Investigación Environmental energy and education policy e3p, "Base de datos panel solar fotovoltaico Universidad Nacional de Colombia, Sede Manizales, Campus la Nubia, Bloque W."

[19] V. Telukunta, J. Pradhan, A. Agrawal, M. Singh, and S. G. Srivani, "Protection challenges under bulk penetration of renewable energy resources in power systems: A review," CSEE J. Power Energy Syst., vol. 3, no. 4, pp. 365-379, Dec. 2017, doi: 10.17775/CSEEJPES.2017.00030.

[20] G. Antonova, M. Nardi, A. Scott, and M. Pesin, "Distributed generation and its impact on power grids and microgrids protection," 2012 65th Annu. Conf. Prot. Relay Eng., pp. 152-161, 2012, doi: 10.1109/CPRE.2012.6201229.

\section{(1) (1) $8 \Theta$}

(C)2021 by the authors. Licensee TESEA, Cartagena, Colombia. This article is an open access article distributed under the terms and conditions of the Creative Commons Attribution (CC BY) license (http://creativecommons.org/licenses/by/4.0/) 